\title{
LICENÇAS SAÚDE DE PROFISSIONAIS DE ENFERMAGEM: CARACTERÍSTICAS E AÇÕES ADOTADAS PELOS ENFERMEIROS-CHEFE*
}

Josimerci Ittavo Lamana Faria: Enfermeira', Paulina Kurcgant ${ }^{2}$

'Enfermeira. Doutora em Enfermagem Fundamental. Faculdade de Medicina de São José do Rio Preto. São José do Rio Preto-SP-Brasil. ${ }^{2}$ Enfermeira. Doutora em Enfermagem. Universidade de Sâo Paulo. São Paulo-SP-Brasil.

RESUMO: Este estudo teve como objetivos compreender a percepção do enfermeiro gerente com relação ao evento Afastamento por Licença Saúde, as ações gerenciais por ele adotadas e o impacto causado por estas ações. A pesquisa de natureza qualitativa teve os dados coletados por meio de entrevista com enfermeiros chefes de quatro unidades de internação de um hospital universitário e foram analisados segundo proposta de análise de conteúdo. Os participantes percebem as licenças saúde como um problema gerencial e as ações gerenciais adotadas frente às licenças saúde objetivaram evitar agravos à saúde do profissional; monitorar as licenças saúde; adequar as escalas de atividades às situações causadas pelo déficit de pessoal; e readaptar os profissionais que retornam das licenças saúde com restrições para o trabalho. Nenhuma mudança efetiva foi considerada nas narrativas dos colaboradores inerente às ações gerenciais adotadas.

DESCRITORES: Equipe de enfermagem; Licença médica; Absenteísmo; Indicadores de qualidade em assistência à saúde; Administração de recursos humanos em hospitais.

\section{NURSING PROFESSIONALS' SICK LEAVE: LICENCIAS DE SALUD DE PROFESIONALES DE CHARACTERISTICS AND ACTIONS ADOPTED BY NURSE MANAGERS*}

ABSTRACT: This study aimed to understand the perception of the nurse manager in relation to the event of Absence from work due to Sick Leave, the managerial actions he adopts, and the impact caused by these actions. This qualitative research involved the Chief Nurses of four inpatient units in a Teaching Hospital. The statements were analyzed using Bardin's proposal for content analysis. The study subjects perceive episodes of sick leave as a managerial problem, and managerial actions are adopted regarding the episodes of sick leave, aiming: to avoid harm to the professional's health; to monitor sick leave; to adapt the schedules of activities to the situations caused by the lack of personnel; and to adjust the duties of those professionals who return from periods of sick leave with restrictions for work. No effective change was considered in the contributors' narratives inherent to the managerial actions adopted.

DESCRIPTORS: Nursing team; Medical leave; Absenteeism; Quality indicators in health care; Administration of human resources in hospitals.

RESUMEN: Este estudio tuvo como objetivos comprender la percepción del enfermero gerente acerca del evento Alejamiento por Licencia de Salud, las acciones administrativas adoptadas por él y el impacto causado por esas acciones. La investigación fue de naturaleza cualitativa y los datos fueron obtenidos por medio de entrevista con enfermeros jefes de cuatro unidades de internación de un hospital universitario y analizados de acuerdo a la propuesta de análisis de contenido. Los participantes perciben las licencias de salud como un problema administrativo y las acciones administrativas adoptadas delante de las licencias de salud tuvieron la finalidad de evitar agravios a la salud del profesional; verificar las licencias de salud; adecuar las escalas de actividades a las situaciones causadas por el déficit de personal; y readaptar los profesionales que vuelven de las licencias de salud con restricciones para el trabajo. No hubo cambio efectivo en las narrativas de los colaboradores acerca de las acciones administrativas adoptadas.

DESCRIPTORES: Equipo de enfermería; Licencia médica; Absentismo; Indicadores de cualidad en asistencia a la salud; Administración de recursos humanos en hospitales.

*Artigo desenvolvido durante o Programa de Pós-Doutoramento na Escola de Enfermagem da Universidade de São Paulo.

Recebido: 13/11/2013

Autor correspondente:

Finalizado: 15/03/2014

Josimerci Ittavo Lamana FariA

Faculdade de Medicina de São José do Rio Preto

Av. Brig. Faria Lima, 5416 - 15090-000 - São José do Rio Preto-SP-Brasil

E-mail: josifaria@famerp.br 


\section{INTRODUÇÃO}

A construção e validação de indicadores para a avaliação de serviços de saúde são de caráter processual e devem ser respaldadas por

referenciais de apoio, sob a ótica dos quais os diferentes elementos constitutivos das estruturas institucionais, dos processos de trabalho e dos resultados da assistência prestada são resgatados e analisados ${ }^{(1: 235)}$.

O conceito de qualidade no contexto da saúde, segundo a Organização Mundial de Saúde ${ }^{(2)}$, constitui-se em um conjunto de atributos que abrange um nível de excelência profissional, o uso eficiente de recursos, um risco mínimo ao usuário e um alto grau de satisfação dos clientes. No âmbito gerencial, os indicadores são adotados como um importante recurso na avaliação e monitoramento da qualidade dos serviços de saúde, direcionando a atenção dos profissionais para resultados específicos, permitindo a construção de cenários que podem facilitar o enfrentamento do futuro organizacional ${ }^{(3)}$.

A adoção de indicadores de qualidade para a avaliação de 4 serviços de saúde pressupõe o reconhecimento da dimensão humana dos profissionais e seu potencial, como elementos imprescindíveis para o alcance das metas institucionais. Portanto, a consideração dos anseios, expectativas e satisfação dos trabalhadores é indispensável na concretização desses propósitos ${ }^{(4)}$. De acordo com essa proposição, a discussão do indicador Afastamento por Licença Saúde (LS) é realizada à luz do referencial de qualidade de vida, que abrange tanto o momento de vida do indivíduo em sociedade, como no trabalho ${ }^{(5)}$.

$\mathrm{Na}$ Enfermagem, o índice de afastamento por LS dos profissionais tem merecido atenção de gestores e dirigentes, pois a realização da assistência de enfermagem com qualidade e segurança, depende, dentre outros fatores de um quantitativo de pessoal ajustado às demandas de cuidados de enfermagem dos pacientes ${ }^{(6)}$.

Legalmente, LS é considerada desde um dia de afastamento do trabalho, até 15 dias. Quando há necessidade de prorrogação, o profissional é submetido à perícia médica, pelo Instituto
Nacional de Seguridade Social (INSS), e a licença passa a ser considerada como licença INSS ${ }^{(6)}$. A ausência de profissionais causada pela LS é classificada como ausência não $\operatorname{prevista}^{(7)} \mathrm{e}$ estudos têm mostrado que é um dos principais componentes deste tipo de ausência ${ }^{(6,8-9)}$.

Embora muitos estudos evidenciem o diagnóstico de altos índices de LS dos trabalhadores de enfermagem, que influenciam a qualidade da gestão de Recursos Humanos $(\mathrm{RH})$ nesta área, na literatura existem lacunas de conhecimento relacionado a esta temática. Diante deste contexto, indaga-se: o que os gestores de enfermagem da área hospitalar têm realizado em relação às LS ocorridas na sua equipe de trabalho? Que resultados foram observados?

O presente estudo teve como objetivos compreender a percepção do enfermeiro gerente com relação ao evento Afastamento por Licença Saúde, as ações gerenciais por ele adotadas e o impacto causado por estas ações.

\section{MÉTODO}

Estudo de caráter qualitativo e de campo foi aprovado pelo Comitê de Ética em Pesquisa em Seres Humanos do Hospital Universitário da Universidade de São Paulo (HU USP), Brasil. (SISNEP/CAAE n.085.0.198.196-10) e desenvolvido nesta mesma instituição. O Departamento de Enfermagem (DE) está subordinado à Superintendência, a qual juntamente com o Conselho Deliberativo constitui a Administração Superior do HU USP. É composto pelas Divisões de Enfermagem Clínica, Cirúrgica e MaternoInfantil e de Pacientes Externos; contém 698 profissionais e 39 vagas para contratação (no período estudado).

Foi iniciado após a identificação das duas unidades de internação com os mais altos índices de afastamento por LS (Clínicas Cirúrgica e Médica) e das duas com os menores (Unidade de Terapia Intensiva de Adultos e Berçário), nos anos de 2009 e 2010. As unidades eram gerenciadas por um enfermeiro e foram eles os convidados a participar deste estudo.

Foram realizadas entrevistas semiestruturadas, com os quatro participantes deste estudo junto às unidades onde atuavam nos meses de fevereiro e março de 2011. As questões norteadoras 
foram: Como você percebe os afastamentos por LS na unidade onde atua? Que ações você tem adotado frente aos afastamentos ocorridos? Quais resultados você percebeu com as ações adotadas? A duração das entrevistas foi em torno de trinta minutos. O conteúdo gravado foi transcrito e organizado resguardando-se o sigilo das informações e anonimato dos participantes. Posteriormente foi submetido à Análise Temática ${ }^{(10)}$.

\section{RESULTADOS}

A análise do conteúdo das narrativas possibilitou a construção de duas categorias, a primeira, A - Afastamentos por LS: visão do gerente de enfermagem, constituída pelas Unidades de Significado (US): As LS: um problema gerencial, Características dos afastamentos por LS e Motivos dos Afastamentos por LS; a segunda, B - Gestão de recursos humanos em enfermagem e os afastamentos por LS, que albergou as US: Ações gerenciais frente às LS: implementação e limites e Impacto causado pelas ações gerenciais frente aos afastamentos por LS.

\section{Afastamentos por LS: visão do gerente de enfermagem.}

Nos depoimentos dos sujeitos deste estudo resgata-se o entendimento dos enfermeiros gerentes acerca da ocorrência de LS, que se destaca como um importante e recorrente problema nas unidades de internação hospitalar, que vem aumentando ao longo dos anos (US - 'As LS: um problema gerencial'):

Os afastamentos têm aumentado, eu percebo. (Enf.1)

Nós temos uma grande quantidade de funcionários que pedem afastamento. (Enf.3)

Isso é um grande problema [...]. (Enf.4)

As falas a seguir referendam a ideia que as frequentes ocorrências destas ausências propiciam o aumento da carga de trabalho dos demais trabalhadores e geram estresse $\mathrm{e}$ sentimentos de insatisfação e não valorização profissional:

Os afastamentos são ruins porque prejudicam a unidade, causam sobrecarga. (Enf.1)

É um momento gerador de estresse para a equipe. (Enf.2)

Quando tem uma situação mais crítica, eles colocam a insatisfação, que não se sentem valorizados e reconhecidos, se sentem sobrecarregados. (Enf.3)

Outra questão resgatada nos discursos é a preocupação relacionada à facilidade que o profissional de saúde tem em obter atestados médicos, o que pode agravar a situação anteriormente descrita além de gerar conflitos, como se verifica nos relatos a seguir:

Porque eles podem pedir o atestado a outros médicos [...] de convênios particulares. E a instituição aceita facilmente. [...] e eu vejo um aumento no número de afastamentos. (Enf.1)

E eu não tenho como contestar [o atestado médico]. (Enf.3)

Também não dá para afirmar [...] não foi por licença realmente médica. (Enf.4)

Os relatos mostram aspectos referentes à imprevisibilidade e ao tempo de duração das licenças: curtas e longas (US - 'Características dos Afastamentos por LS'). As LS curtas, muitas vezes estão relacionadas às necessidades rotina com sua saúde e, as longas, ao exercício laboral e à qualidade de vida, como se verifica a seguir:

O difícil é que, estas licenças, não são previstas [...]. Oscila muito [...]. As licenças de poucos dias ou até de 15 dias, algumas, são relacionadas ao próprio profissional, como para tratamento odontológico. Outras são relacionadas à carga de trabalho [...]. (Enf.4) 
As licenças de longa permanência, as INSS, vem se agravando pela condição de vida do indivíduo. (Enf.2)

A maior frequência de LS entre os profissionais de nível médio e faixa etária intermediária é identificada nos depoimentos:

Temos um quadro muito bom de enfermeiros [...] quem sai [LS] são os funcionários do nível médio. (Enf.4)

Tenho funcionário com mais de 50 anos. Mas não são eles que pegam mais atestados, são os funcionários mais jovens. (Enf.3)

A carga de trabalho é observada nos relatos como fator de grande impacto no adoecimento dos trabalhadores de enfermagem (US - 'Motivos dos Afastamentos por $\mathrm{LS}^{\prime}$ ):

Muitas vezes ele sai $[\mathrm{LS}]$ por uma dor, na região dorsal, dor no tornozelo, dor nas costas. É da assistência mesmo que ele faz aqui. (Enf.4)

Atualmente, tenho mais afastamentos por motivos físicos, mas o psicológico também está incluído. (Enf.2)

Os depoimentos revelam motivos que impactam na carga de trabalho dos profissionais de enfermagem, como o aumento da expectativa de vida e o aumento do número de trabalhadores atuantes com restrição de saúde para o trabalho:

Estão mais envelhecidos e demandam uma assistência de enfermagem muito maior. (Enf.2)

Destes funcionários com restrição [...] a gente ajuda bastante no sentido de não deixar fazer esforço [...]. Mas aí tem o outro [que não tem restrição] que tem uma sobrecarga. (Enf. 4)

Outros motivos identificados se voltam à condição de vida e falta de cuidado com a saúde e, à frequente necessidade de alguns profissionais se afastarem por LS:
Hoje, a maioria trabalha fora [...] algumas tem filhos adolescentes com problemas com drogas e violência. [...] isso gera um desgaste muito grande. (Enf. 2)

Muitos têm dupla jornada, filhos, o trabalho em casa. (Enf. 3)

\section{Gestão de recursos humanos em enfermagem e os afastamentos por LS}

Ações que visam à prevenção de danos e/ou agravos à saúde do profissional são identificadas nos seus relatos dos enfermeiros gerentes (US 'Ações gerenciais frente às LS: implementação e limites'):

Vimos junto à administração para providenciar equipamentos [...] desta forma nós tiramos a carga física do funcionário. (Enf.2)

O que sempre peço a eles é que não façam atividades físicas sozinhos. (Enf.3)

É utilizado o transfer para ajudar a tirar o paciente do leito. (Enf.4)

Outra ação resgatada nos relatos é a dispensa do funcionário do serviço:

Às vezes eles passam pelo médico e ele não dá a licença. Então o que eu tenho que fazer é mandálo embora [...] dou a folga [...]. (Enf.3)

Medidas rotineiras relacionadas à reorganização das escalas, diária e mensal, à realização de horas extras, à priorização dos cuidados essenciais e ao monitoramento dos afastamentos por intermédio do diálogo com o funcionário são identificadas nos depoimentos:

Às vezes também, já sabendo, por exemplo, que faltará funcionário no turno da tarde, nós fazemos todas as prescrições de manhã. (Enf.1)

Reorganizo o cuidado de acordo com a equipe 
que disponho em nível local e vejo em outras unidades, se consigo algum recurso. (Enf.2)

Tento conversar 'Por que você saiu de licença, o que aconteceu?'. (Enf.4)

Ações adotadas em relação às LS de longa duração são voltadas à comunicação e busca de soluções junto ao nível hierárquico superior:

Quando tenho problemas, comunico à diretora responsável pela divisão [...]. (Enf.3)

Temos mostrado o aumento da taxa de ocupação, que a complexidade tem aumentado, para ver se conseguimos uma adequação melhor frente a esses afastamentos. (Enf.1)

Ações para a readaptação dos profissionais que retornam das LS com restrições físicas e/ou psíquicas para o trabalho são identificadas nas narrativas:

Muitos retornam com o aval da psiquiatria de que [...] fazer as suas atividades, só que você [...] tem a visão de que ele não tem condições de exercer essas atividades. (Enf.2)

Os pacientes de alta dependência ficam sob o cuidado do restante da equipe. (Enf.3)

Outro enfoque que compõe a US - 'As ações gerenciais frente às LS: implementação e limites' diz respeito aos limites da autonomia do gerente de enfermagem para a resolução do fenômeno afastamento por LS. Ele defende a importância da existência de programas/ serviços de prevenção e acompanhamento do trabalhador, mas entende que não tem autonomia para estabelecer mudanças:

Hojetemos dificuldadeemrealizarencaminhamentos para acompanhamento psicológico. Isso dificulta muito porque ficamos com a batata quente na mão. (Enf.2)

Houve um tempo que podíamos fazer contratações temporárias [...]. (Enf.1)
Quanto aos resultados advindos das ações gerenciais implementadas pelos enfermeiros gerentes frente ao evento Afastamento por LS, US - 'Impacto das ações gerenciais frente aos afastamentos por $\mathrm{LS}^{\prime}$, observa-se nos depoimentos que as medidas adotadas levam à situações conflitantes e estressantes, além de sobrecarregar os profissionais presentes na unidade:

Nem todos se sentem aptos a fazer o que é específico de cada unidade. [...] se sente assim, inseguro. (Enf.1)

Acabamos sobrecarregando o outro [...]. E aí, pode ser que, por consequência, ele também saia de licença no futuro, ou um pouco mais próximo. (Enf.4)

Observa-se que no entendimento dos participantes do estudo, que não houve impacto positivo no índice de afastamento por LS, pois não diminuiu, ao contrário, está aumentando, e a insatisfação dos profissionais continua presente, como ilustra a narrativa:

Quanto ao impacto resultado dessas ações, não vejo muita mudança, continuam os afastamentos, a sobrecarga de trabalho, a insatisfação. (Enf.1)

\section{DISCUSSÃO}

As estatísticas atuais refletem dados preocupantes acerca de doenças relacionadas às atividades laborais na enfermagem. A frequente exposição dos profissionais às cargas de trabalho desencadeia processos de desgaste que comprometem a sua saúde e a sua qualidade de vida, sinalizando a necessidade de intervenções nessa realidade ${ }^{(11)}$.

Estudo realizado no HU-USP, mesmo cenário da presente pesquisa, mostrou um crescente aumento do número de LS durante o período de 2003 a 2007. A quantidade de dias perdidos por LS triplicou quando comparado ao ano de 2003 apresentando percentuais de aumento diferentes entre as categorias profissionais ${ }^{(8)}$.

Pesquisas buscam correlacionar evidências entre características individuais e organizacionais ${ }^{(12-13)}$, como más condições de trabalho ${ }^{(13)}$, desgastes 
físicos e psíquicos ${ }^{(11)}$ que contribuem para o absenteísmo que, por sua vez integra os afastamentos por LS na equipe de enfermagem. Um destes estudos ${ }^{(12)}$ revela fatores relacionados ao absenteísmo como: a coesão do grupo e delegação de autonomia inadequadas, ambiguidade de funções, rotina de trabalho ineficaz e o efeito da carga de trabalho no ambiente profissional.

Pesquisa realizada no mesmo lócus deste estudo mostrou que, em relação ao quadro de enfermagem, os profissionais de nível médio responderam por $80,1 \%$ das $L S^{(14)}$. Outras pesquisas corroboram estes resultados ${ }^{(8,13,15)}$, e indicam a provável associação das LS com as atividades profissionais, em especial, na assistência direta ao paciente. Quanto à faixa etária, estudo ${ }^{(14)}$ concluiu que a idade não condiciona as ausências por LS.

As condições de trabalho causam sofrimento físico e mental no trabalhador ${ }^{(16)}$. Estudo realizado em hospitais brasileiros acerca da articulação entre os processos de desgaste e as formas de trabalho, por meio da exposição dos trabalhadores às cargas psíquicas no desempenho de suas atividades, evidenciou que "as cargas psíquicas além de serem atribuídas a algumas condições de trabalho, são potencializadoras e potencializadas pela maioria das outras cargas"(11:7).

A relação linear entre o aumento da carga de trabalho e o aumento de absenteísmo foi encontrada em pesquisa realizadae, os autores explicitam que a sobrecarga de trabalho pode contribuir, significativamente, para os problemas de saúde ocupacional ${ }^{(17)}$. Pesquisa ${ }^{(14)}$ evidencia que o índice mensal de absenteísmo por doença foi inversamente proporcional à taxa de ocupação, ou seja, os profissionais suportam a carga mais intensa em períodos em que o ritmo da assistência é mais acelerado e, após, muitos entram em licença saúde pelo consequente adoecimento $^{(14)}$.

Pelo fato do quadro de pessoal de enfermagem ser composto, principalmente, por pessoas do sexo feminino, é importante sublinhar o desdobramento da jornada do trabalho inerente a este gênero, em função das tarefas domésticas que acontecem, sem descanso semanal ou férias remuneradas, configurando um aspecto negativo para a autoestima, que podem levar à fadiga crônica e à exaustão física e mental. A culpa por não conseguir oferecer atenção aos filhos, em detrimento do trabalho, é outro desgaste sofrido, em especial, pelas mulheres, que desencadeiam sintomas psicossomáticos e comprometimento da sua qualidade de vida e familiares próximos ${ }^{(11)}$. Desta forma, para muitas enfermeiras, qualidade de vida depende do equilíbrio entre trabalho e vida doméstica ${ }^{(18)}$.

Uma das questões que interfere, na carga de trabalho nas unidades de internação e na qualidade de vida do funcionário, é o quadro de pessoal insuficiente. O impacto, na equipe de enfermagem, causado pelos afastamentos devido às LS, de curta ou longa duração, é fato indiscutível e referendado por muitos estudiosos sobre o absenteísmo-doença ${ }^{(8,13-14)}$.

A composição do quadro de pessoal de enfermagem atuante nos serviços de saúde, com profissionais que não podem realizar, de forma plena, as suas atribuições, acarreta sobrecarga de trabalho aos colegas de equipe, em especial, àqueles que têm a condição física e psíquica aparentemente preservada. Os desdobramentos podem repercutir em faltas e licenças médicas devido à sobrecarga sofrida ${ }^{(9)}$.

Em relação às ações adotadas devido às ausências por LS, dois aspectos distintos são importantes para o processo gerencial do fenômeno LS: o primeiro diz respeito à frequência dos afastamentos, que determina a necessidade de aplicação de medidas gerencias de imediato, como a redistribuição das atividades assistências diárias entre os profissionais presentes, entre outras. O segundo, inerente aos afastamentos de longa duração, exige ações que envolvem negociação com enfermeiros gerentes de outras unidades e/ou com superiores, para cobrir o período de licença ou efetuar uma contratação temporária ${ }^{(8)}$. O impacto das ações rotineiras adotadas frente às situações geradas pela ausência do profissional na unidade de internação devido às LS e à volta do profissional das LS, com restrições para o trabalho levou, em seu conjunto, à sobrecarga de trabalho, à insegurança, ao conflito e às situações estressantes, que repercutem em insatisfação na equipe de enfermagem.

Diante destas assertivas é importante lembrar que existe íntima relação entre a satisfação ou insatisfação profissional e os afastamentos por LS, ambas apontadas como indicadores de qualidade no gerenciamento de recursos humanos em enfermagem ${ }^{(1)}$. O ambiente de trabalho cada vez mais estressante possibilita o aumento das 
LS, o que proporciona mais trabalho para quem está presente e consequente sobrecarga, que por sua vez eleva o estresse, que pode contribuir para a ocorrência de mais afastamentos por LS. Esta situação faz lembrar à descrita em estudo com a denominação de 'Ciclo de Escassez de Profissionais de Enfermagem" ${ }^{\prime(19)}$.

As más condições do trabalho de enfermagem e o impacto na qualidade de vida dos profissionais de enfermagem são amplamente conhecidos. Embora alvo de preocupações de gestores de serviços de saúde e estudiosos desta temática, as intervenções são tímidas e não repercutem em transformação desta realidade. Cabe ressaltar

se o meio de trabalho e o produto desse mesmo trabalho é o ser humano, que sofre, se desgasta, adoece e morre, então ele deve ser a razão pela qual o serviço se estrutura - tanto nos aspectos físicos quanto nas relações pessoais e hierárquicas ${ }^{(11: 7)}$.

Estudos que evidenciem as ações que causam impacto positivo no índice de LS necessitam ser desenvolvidos. É importante destacar a necessidade, por parte dos profissionais de enfermagem, de refletir sobre a situação posta, e criar espaços para maior participação nas políticas de recursos humanos nas instituições de saúde.

O fenômeno afastamento por LS, multifacetado e complexo, gera situações de difícil solução. O controle do afastamento por LS e suas consequências, assim como o aumento da satisfação no trabalho, somente terão êxito com o desenvolvimento de estratégias multidisciplinares e abrangentes que levem em conta as condições físicas, psicológicas e psicossociais do trabalho ${ }^{(17)}$.

\section{CONSIDERAÇÕES FINAIS}

Na percepção dos enfermeiros gerentes, o complexo fenômeno Afastamento por LS se constitui em um sério problema gerencial associado às condições de trabalho, em especial às cargas de trabalho, que implicam no adoecimento dos profissionais, principalmente os de nível médio.

As ações gerenciais, adotadas pelos enfermeiros, frente aos afastamentos por LS se voltaram à orientação para minimizar a carga física de trabalho e evitar agravos à saúde do profissional relacionado ao processo de trabalho; ao monitoramento das LS; à cobertura das ausências devido à LS com solicitação de remanejamento de funcionário ou plantão extra e à priorização de cuidados a serem prestados aos pacientes, entre outras. Ações realizadas para readaptação dos profissionais que retornam das LS com restrições para o trabalho abrangeram a orientação e supervisão das atividades laborais e a conscientização da equipe de enfermagem sobre a situação.

As ações gerenciais adotadas quanto ao déficit no quadro de pessoal, provocado pela ausência do profissional na unidade de trabalho, resultaram em sobrecarga de trabalho, aumento do estresse e insatisfação do profissional, aspectos estes que levam à má qualidade de vida no trabalho e que podem contribuir para o aumento das LS e consequente queda na qualidade da assistência.

Não foi identificado impacto positivo, ou seja, nenhuma mudança efetiva foi considerada nas narrativas dos colaboradores do estudo, pois os índices, a despeito das ações adotadas, continuam subindo ano a ano. Estes achados mostram urgência na reivindicação de mudanças nas Políticas de Recursos Humanos em Saúde para que levem em consideração o perfil e as necessidades dos trabalhadores de Enfermagem com propostas de impacto para a melhoria da qualidade de vida dos profissionais de enfermagem.

Este estudo contribui para o conhecimento na área de indicadores de qualidade na gestão de RH em Enfermagem, em especial, no que se refere ao fenômeno Afastamentos por LS. Propiciou a identificação de ações gerenciais adotas por enfermeiros frente às LS e o impacto causado por elas. Acredita-se que outros estudos devem ser realizados sobre esta temática, buscando melhor compreensão do fenômeno e das ações gerenciais que levem à diminuição do índice de afastamento por LS, possibilitando o avanço da prática gerencial baseada em evidências.

\section{REFERÊNCIAS}

1. Lima AFC, Kurcgant P. Indicadores de qualidade no gerenciamento de recursos humanos em enfermagem. Rev. bras. enferm. 2009;62(2):234-9.

2. Organização Mundial da Saúde (OMS). Avaliação dos programas de saúde: normas fundamentais para a sua 
aplicação no processo de gestão para o desenvolvimento nacional na saúde. Genebra: OMS; 1981.

3. Bittar OJN. Indicadores de qualidade e quantidade em saúde. Rev. adm. saúde. 2004;6(22):15-8.

4. Leitão RER, Kurcgant P. Qualidade na prática gerencial de Enfermagem. Niterói: Intertexto; 2004.

5. Kurcgant $P$, Tronchin DMR, Melleiro MM, Castilho V, Machado VB, Pinhel I, et al. Indicadores de qualidade e a avaliação do gerenciamento de recursos humanos em saúde. Rev Esc Enferm USP. 2009;42(n.esp):116873. Disponível: http://dx.doi.org/10.1590/S008062342009000600004.

6. Laus AM, Anselmi ML. Ausências dos trabalhadores de enfermagem em um Hospital Escola. Rev Esc Enferm USP. 2008; 42(4):681-9. Disponível: http://dx.doi. org/10.1590/S0080-62342008000400010

7. Gaidzinski RR, Lima AFC, Soares AVN, Fugulin FMT, Sancinetti TR, Castilho V. Gestão de pessoal de enfermagem e o sistema de assistência de enfermagem. In: Gaidzinki RR. Diagnóstico de enfermagem na prática clínica. Porto Alegre: Artmed; 2008. p. 47-61.

8. Gaidzinski RR, Fugulin FMT, Castilho VC. Dimensionamento de pessoal de enfermagem em instituições de saúde In: Kurcgant $\mathrm{P}$, coordenadora. Gerenciamento em enfermagem. Rio de Janeiro: Guanabara Koogan; 2005. p. 125-37.

9. Estorce TP, Kurcgant P. Licença médica e gerenciamento de pessoal de enfermagem. Rev Esc Enferm USP. 2011;45(5):1199-205. Disponível: http://dx.doi. org/10.1590/S0080-62342011000500024

10. Campos EC, Juliani CMCM, Palhares VC. O absenteísmo da equipe de enfermagem em unidade de pronto socorro de um hospital universitário. Rev. Eletr. Enf. [Internet]. 2009;11(2):295-302. Disponível: http://www.fen.ufg.br/revista/v11/n2/v11n2a09.htm

11. Felli VE, Tronchin DMR. A qualidade de vida no trabalho e a saúde do trabalhador de enfermagem. In: Kurcgant P, coordenadora. Gerenciamento em enfermagem. Rio de Janeiro: Guanabara Koogan; 2005. p. 90-107.

12. Ministério da Saúde (BR). Conselho Nacional de Saúde. Diretrizes e Normas regulamentadoras de pesquisa envolvendo seres humanos. Resolução n. 196, de 10 de outubro de 1996. Brasília; 1996. [Internet] [acesso em 23 fev 2013]. Disponível: http://conselho.saude. gov.br/resoluções/reso_96htm

13. Bardin L. Análise de conteúdo. São Paulo: Pinheiro; 2011.

14. Mininel VA, Baptista PCP, Felli VEA. Cargas psíquicas e processos de desgaste em trabalhadores de enfermagem de hospitais universitários brasileiros. Rev. Latino-Am. Enferm. 2011;19(2):[09 telas].

15. Nyathi MA, Jooste K. Working conditions that contribute to absenteeism among nurses in a provincial hospital in the Limpopo Province. Curationis. 2008;31(1):28-37.

16. Silva DMPP, Marziale MHP. Condições de trabalho versus absenteísmo-doença no trabalho de enfermagem. Ciênc. cuid. saúde. 2006;5(n.supl):166-72.

17. Sancinetti TR, Gaidzinski RR, Felli VEA, Fugulin MFT, Baptista PCP, Cianpone MHT et al. Absenteísmodoença na equipe de enfermagem: relação com a taxa de ocupação. Rev Esc Enferm USP. 2009;43(n. esp2):1277-83. Disponível: http://dx.doi.org/10.1590/ S0080-62342009000600023

18. Barboza DB, Soler ZASG. Afastamentos do trabalho na enfermagem: ocorrências com trabalhadores de um Hospital de Ensino. Rev. Latino-Am. Enfermagem. 2003;11(2):177-83.

19. Duarte MLC, Avelhaneda JPRR. A saúde do trabalhador na estratégia de saúde da família: percepções da equipe de enfermagem. Cogitare enferm. 2013;18(2):323-30.

20. Sápia T, Felli VEA, Ciampone MHT. Problemas de saúde de trabalhadores de enfermagem em ambulatórios pela exposição à cargas fisiológicas. Acta Paul. Enferm. 2009;22(6):808-13.

21. Rauhala A, Kkivimaki $M$, Fageström $L$, Elovainio M, Virtanen M, Vahtera J, et al. What degree of work overload is likely to cause increased sickness absenteeism amomg nurses? Evidence from the RAFAELA patient classification system. J. adv. nurs. 2007;57(3):286-95.

22. Alves M, Godoy SCB, Santana DM. Motivos de licenças médicas em um hospital de urgênciaemergência. Rev. bras. enferm. 2006;59(2):195-200.

23. Greene K. Introducing contemporary shift patterns in a hospice setting. Nurs. stand. 2007;21(27):14-20.

24. Rajbhandary S, Basu K. Working conditions of nurses and absenteeism: is there a relationship? An empirical analysis using National Survey of the Work and Health of Nurses. Health policy. 2010;97(2):152-9.

25. Gaudine A, Gregory C. The accuracy of nurses' estimates of their absenteeism. Journal of Nursing Management. 2010;18:599-605. 